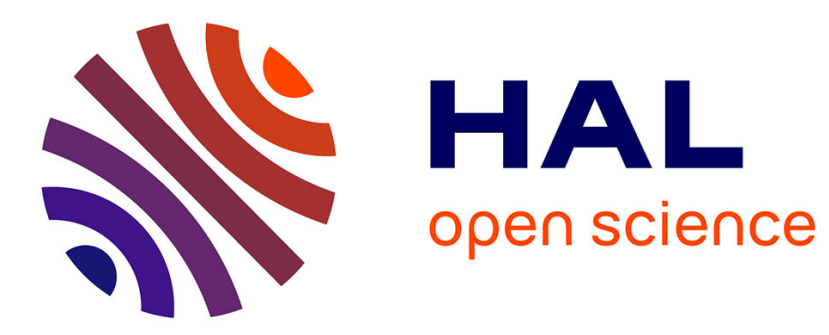

\title{
Vygotski aujourd'hui en management
}

Valérie-Inès de La Ville, Bernard Leca, Jean-Louis Magakian

\section{To cite this version:}

Valérie-Inès de La Ville, Bernard Leca, Jean-Louis Magakian. Vygotski aujourd'hui en management. Revue management \& avenir, 2011, Vol. 2 ( $\left.\mathrm{N}^{\circ} 42\right)$, p. 78-88. 10.3917/mav.042.0078 . hal-00718621

\section{HAL Id: hal-00718621 \\ https://hal.science/hal-00718621}

Submitted on 14 Mar 2019

HAL is a multi-disciplinary open access archive for the deposit and dissemination of scientific research documents, whether they are published or not. The documents may come from teaching and research institutions in France or abroad, or from public or private research centers.
L'archive ouverte pluridisciplinaire HAL, est destinée au dépôt et à la diffusion de documents scientifiques de niveau recherche, publiés ou non, émanant des établissements d'enseignement et de recherche français ou étrangers, des laboratoires publics ou privés. 


\title{
Vygotski aujourd'hui en management
}

\author{
par Valérie-Inès de La Ville ${ }^{1}$, \\ Bernard Leca 2 \\ Jean-Louis Magakian 3
}

1 Professeur Des Universités, Directrice du Centre Européen des Produits de l'Enfant, IAE - Université de Poitiers, Laboratoire Centre de Recherche en Gestion EA 1722, delaville@iae.univ-poitiers.fr

2 Professeur Associé, Rouen Business School, blc@rouenbs.fr

3 Enseignant-Chercheur ESC Saint-Etienne, jean-louis_magakian@esc-saint-etienne.fr

Pourquoi proposer aujourd'hui une série d'articles sur l'influence de Lev Vygotski en management?

L'œuvre de ce psychologue n'est pas neuve en soi. Vygotski est né en 1896 et mort en 1934. II n'est pas non plus inconnu. Malgré une œuvre empirique qui ne s'étale que sur une dizaine d'années, Vygotski est reconnu depuis les années 30 comme l'un des chercheurs les plus importants du 20ème siècle en psychologie et en pédagogie au travers de la Cultural-Historical Activity Theory (CHAT) dont il est le fondateur. Cette approche a donné lieu à un vaste héritage regroupé parfois sous le terme de théorie de l'activité (TA).

Bien que différentes disciplines du management aient depuis des décennies mobilisé, directement ou par le biais de ses épigones, les principaux acquis de l'œuvre de Vygotski, de nouveaux questionnements théoriques en management s'en inspirent directement. Ces approches permettent d'envisager des dévelop- pements importants pour la recherche en sciences de gestion, dont il est aujourd'hui possible d'apercevoir les prémices.

A cette fin, la Rouen Business School a organisé deux ateliers de recherche visant à faire le point sur les apports actuels de Vygotski dans les différents domaines des sciences de gestion. A la suite de ces rencontres, nous avons décidé de contacter d'autres collègues s'inspirant de cette œuvre ou se situant dans sa filiation directe, afin de proposer ce dossier à la revue Management \& Avenir. Le dossier présenté est ainsi le résultat des échanges ayant eu lieu lors de ces ateliers, des textes que nous ont fait parvenir nos 
collègues, ainsi que des commentaires et demandes de révision des chercheurs - français et étrangers - qui ont apporté leur contribution pour améliorer la qualité scientifique des textes finalement retenus.

Le message général que ce dossier souhaite porter est relativement simple: au sein des approches centrées sur 'les pratiques du management' qui se sont développées depuis quelques années, les travaux mobilisant les acquis de la TA constituent un ensemble cohérent et flexible.

Ce cadre épistémologique et théorique offre aux chercheurs en sciences de gestion de solides fondements conceptuels et des voies d'innovation méthodologique ouvrant un dialogue approfondi avec d'autres démarches relativement proches comme le pragmatisme, le réalisme critique ou encore la théorie de l'acteur réseau. Les différents articles réunis dans ce numéro spécial ainsi que l'entretien mené avec Yrjö Engeström, présentent quelques exemples de recherches récentes menées dans différentes disciplines des sciences de gestion et situent les apports que l'on peut en espérer pour parvenir à une compréhension approfondie du management en train de se faire... Mais avant $d^{\prime}$ inviter le lecteur à se plonger dans ces recherches originales, nous proposons un bref exposé introductif sur la réception progressive des travaux de Vygotski par la communauté des chercheurs en management, afin d'éclairer l'état actuel de son champ d'influence et entrevoir les perspectives qui se dessinent pour l'avenir.

\section{Vygotski hier}

Dans un des premiers recueils d'articles de et sur Vygotski publié en français, J.P. Bronckart (1985), auquel nous empruntons en partie le titre de ce dossier, considère que deux raisons principales expliquent pourquoi une œuvre aussi importante n'a été exploitée que de façon tardive par la communauté de recherche francophone. D'une part, les textes originaux de Vygotski n'ont été que partiellement traduits en anglais et parfois de façon assez approximative, et ce n'est que très récemment qu'un travail fidèle de traduction a été mené en français (Vygotski 1997 ; Clot 1999). D'autre part en raison d'une surexposition des apports du psychologue russe dans le domaine de la pédagogie, ce qui a conduit à restreindre la sphère d'influence de sa pensée à des enjeux d'éducation et de formation. A cela s'ajoute la proximité de ses domaines de recherches avec ceux que Jean Piaget a également abordés et qui ont profondément influencé la communauté de recherche francophone. Les 
démarches d'investigation de ces deux psychologues s'appuient sur des fondements épistémologiques distincts : les travaux de Piaget reflètent I'influence de Kant et notamment du processus de mentalisation des idées, du dualisme cartésien de la séparation du corps et de l'intellect, et de l'hypothèse évolutionniste des stades cognitifs; ceux de Vygotski reflètent la triple inspiration de Hegel et la logique dialectique, le monisme de Spinoza et donc l'unité de la conscience et du corps, et du matérialisme de Marx avec en particulier l'ancrage historique des activités humaines. On retrouve cette triple influence dans l'ouvrage central de Vygotski, Pensée et Langage (1997). Vygotski a entraîné dans son sillage plusieurs disciples, lesquels approfondiront et développeront les fondements de la TA : Leontiev (1978) a construit l'appareillage théorique permettant de distinguer l'activité des actions, et Luria (1976) a détaillé l'usage des outils culturels dans les activités cognitives. Les travaux de Bakhtine sont également associés à ce courant dit de la 'psychologie soviétique', notamment en raison de la proximité de sa notion de 'dialogisme' qui s'apparente aux hypothèses vygotskiennes relatives au langage : I'influence de I'intertextualité et des mots en tant qu'outils culturels. Au-delà de ces développements théoriques, évoqués de façon trop rapide dans cette introduction, une seconde vague de travaux plus contemporains cherche à articuler les concepts élaborés par Vygotski avec d'autres domaines des sciences sociales, ce qui permet d'ouvrir de nouvelles pistes prometteuses. Que ce soit dans le domaine de l'anthropologie cognitive (Donald, 1991 ; Norman, 1993), de la psychologie sociale (Bruner, 1990 ; Wertsch, 1985) ou du constructionnisme social où Kenneth Gergen (1999) pousse encore plus loin les principes interactionnistes pour formaliser un courant radical dans lequel le sujet et sa vision du monde dépendent étroitement des usages collaboratifs et sociaux du langage. Parallèlement, d'autres travaux, qui s'attachent à utiliser les concepts vygotskiens de façon plus opératoire, peuvent être scindés en deux grands ensembles. Le premier s'intéresse aux contextes d'interaction sociale, et regroupe notamment la cognition située (Suchman, 1987), la cognition distribuée (Hutchins, 1995), les contextes discursifs (Shotter, 1993), les interactions avec les contextes technologiques (Nardi, 1996), ainsi que les communautés de pratiques (Lave \& Wenger, 1991). Le second comprend les approches cliniques des interactions en situation de travail telles que les développements de la Théorie de l'Activité (Engeström, 1987), la clinique de l'activité (Clot, 1999) ou encore la démarche « Fifth Dimension» (Cole, 2006) qui s'intéresse aux processus d'apprentissage. 


\section{Vygostki aujourd'hui en management}

En management la TA est utilisée dans plusieurs disciplines. Par exemple, au niveau de l'étude des organisations, Spender reprend ce cadre d'analyse pour donner une dimension organisationnelle aux questions portant sur la cognition collective en tant qu'activité (1995), la mémoire organisationnelle (1996), ou encore de la formation des connaissances collectives (1998). Blackler fait de son côté le lien entre les contextes organisationnels et le management des connaissances dans sa dimension sociale $(1993$; 1995), et par la suite la relation entre les processus 'organisant' et les organisations complexes (2000). Dans une approche conversationnelle et discursive de la cognition, puisant directement dans l'œuvre de Vygotski l'idée de la créativité du langage, Cunliffe (2001a et b ; 2002) et Shotter \& Cunliffe (2003) considèrent que les managers (dirigeants ou intermédiaires) comme autant d'auteurs, de romanciers (novelists), capables de créer entre eux et autour d'eux des territoires discursifs dialogiques engageant la construction du sens collectif tout comme la construction de la perception de soi. Lorino pour sa part, utilise Vygotski dans le cadre de la mesure des activités, mettant en relation les apports de la TA notamment dans l'usage médiatisant des instruments de contrôle avec les actions menées par l'organisation (1995). Plus récemment, Jarzakbowski ouvre le chantier de la stratégie en pratique en s'appuyant directement sur la théorie de l'activité, mettant en évidence plusieurs formes d'activités situées permettant de construire la stratégie (2003 ; 2005 ; 2010).

\section{Vygotski demain}

La réception en management de l'œuvre de Vygotski et des développements qu'elle a inspirés, apparaît comme sélective. L'intérêt de la TA pour les processus d'interaction sociale médiatisés par les outils, et donc de l'appropriation des pratiques managériales à partir de la maîtrise des outils de gestion, ont été reçus relativement tôt, notamment dans des domaines comme les systèmes d'information (Nardi, 1996; Suchman, 1987) et le contrôle de gestion (Laguecir, 2003 ; Lorino, 2006) où les chercheurs se sont rapidement interrogés sur l'impact des artefacts techniques sur les activités cognitives des managers. D'autres aspects de l'œuvre de Vygotski ont cependant reçu une réception plus incertaine... C'est notamment le cas en ce qui concerne les démarches processuelles. Dans le champ des sciences de gestion, les processus 
sont en effet considérés comme se déployant temporellement et sont conceptualisés comme répondant à une logique d'accumulation progressive. Les processus de changement organisationnel ou institutionnel (Greenwood, Suddaby et Hinings, 2002) constituent des exemples parmi d'autres de cette approche. En se fondant sur la TA, Blackler et Regan $(2006 ; 2009)$ ont montré que les processus de changement ne sont en réalité ni clairement délimités, ni linéaires. Ces chercheurs soulignent que les processus d'accumulation successive sont rares et qu'il existe bien plus fréquemment des confusions, des incompréhensions, des faux départs ou des voies sans issues... En se référant aux apports de la TA, ces auteurs montrent également que l'échec dans la diffusion d'une pratique peut venir, non pas de la capacité à internaliser cette pratique, mais bien de l'incapacité à l'externaliser dans le champ des pratiques collectives qui constituent l'organisation (Schatzki, 2001 ; 2005). Ces travaux insistent sur la nécessité de dépasser une vision purement interprétative des processus pour les ancrer dans des contextes matériels, en prenant en compte la singularité historique des acteurs, des situations organisationnelles, des techniques et outils disponibles ainsi que de la complexité des interactions qui en résultent. Si ce cadre conceptuel connaît un certain intérêt, il semble cependant qu'il soit demeuré marginal dans les études processuelles (de La Ville 1996).

Notons également l'influence des travaux de Vygotski pour les travaux se situant autour de l'approche 'stratégie comme pratique' (e.g. Gorlsorkhi, 2006 ; Gorlsorkhi, Rouleau, Seidl et Vaara, 2010) et notamment des travaux de Paula Jarzabowski. Alors que les auteurs se réclamant des approches par les pratiques mobilisent un grand nombre d'approches parfois disparates, Jarzabowski $(2005,2010)$ insiste sur l'apport très particulier de la TA. C'est du reste cette spécificité que souligne également Yrjö Engström dans l'entretien qu'il a accordé à Jean Louis Magakian dans le cadre de ce dossier et dans lequel il exprime clairement ses réserves vis-à-vis de la diversité des théories mobilisées dans les approches dites de la pratique. Dans cette 'soupe' - pour reprendre le terme d'Engeström - que composent les approches de la 'pratique managériale', la théorie de l'activité à travers ses extensions, propose un angle, des concepts et un intérêt particulier.

Celui-ci tient d'abord à ce que la méthodologie de la TA cherche à faire l'effort de prendre en compte le substrat matériel de l'activité et ne se satisfait pas d'une interaction - même complexe - entre la structure et l'agence. En restant arc-boutée sur ce niveau d'analyse particulièrement fin, elle évite les 
débats relativement stériles sur les relations entre structure et agence qui tendent à obscurcir de nombreuses discussions relatives aux 'pratiques managériales'. L'article de Magakian dans ce dossier montre bien comment une approche par les activités permet une " montée en généralité » progressive de I'analyse, et évite de partir avec un bagage théorique particulièrement lourd, qui risquerait d'étouffer le terrain en lui surimposant des grilles de lecture arrêtées a priori.

L'intérêt de se concentrer sur les activités des acteurs commence à influencer également d'autres courants de recherche comme la Théorie NéoInstitutionnaliste (TNI). Alors que ce courant s'est traditionnellement attaché à démontrer l'influence des structures sur les acteurs (Meyer et Jepperson, 2000 ; Meyer et Rowan, 1977 ; Scott, 2001), et les relations entre agence et structure, la TNI s'intéresse de manière croissante aux origines micro-sociales des processus institutionnels (Powell et Colyvas, 2008) comme aux activités ordinaires des acteurs. La notion de travail institutionnel récemment développée (Benslimane et Leca, 2010 ; Lawrence et Suddaby, 2006) vise précisément à rendre compte de l'activité des différents protagonistes engagés dans des processus institutionnels. Le lien avec les travaux fondateurs de Vygotski apparaît alors évident et pourrait permettre de mieux analyser les comportements des acteurs et d'approfondir la compréhension des processus d'analyse et d'apprentissage des agents que la plupart des travaux néoinstitutionnalistes actuels ont eu tendance à ignorer. Se posent cependant d'importantes questions et pistes de recherche au nombre desquelles celle de déterminer comment combiner une approche dualiste comme la TNI qui distingue les institutions d'une part de l'agentivité (agency) des acteurs d'autre part, avec l'approche moniste de la TA qui articule le monde social autour de l'activité et suggère que l'activité se fonde sur un engagement réflexif. Or, la TNI considère également que les acteurs ont pafrois des pratiques institutionnalisées qui sont tellement tenues pour acquises qu'elles s'imposent comme naturelles à ces acteurs qui ne songent pas à les remettre en cause (Huault et Leca, 2009). Comment alors penser les modalités d'interaction entre ces deux approches ? Ceci reste encore largement à définir.

Par ailleurs, la TA constitue en soi, et indépendamment des autres courants de recherche qu'elle enrichit, une méthodologie importante dans l'éventail théorique d'analyse des organisations. En tant que telle, elle semble particulièrement bien adaptée pour rendre compte de nouveaux contextes organisationnels, comme les organisations collaboratives, les communautés 
ouvertes, pour analyser les interactions sociales avec les, et par l'intermédiaire des outils nés de l'informatique. La place croissante de nouveaux dispositifs socio-techniques dans les interactions sociales permet d'envisager des investigations originales et novatrices. Ainsi, c'est la théorie de l'activité que Nardi (2010) utilise pour rendre compte de la multiplicité des interactions entre acteurs dans les jeux dits " massivement multi- joueurs ", où les sujets s'immergent par le biais d'interfaces informatiques pour rendre compte des interactions entre les joueurs et la machine, mais aussi entre les joueurs entre eux.

\section{Les articles de ce dossier}

Les quatre premiers articles de ce dossier entreprennent de montrer, dans des domaines différents de la gestion, GRH, Marketing, contrôle de gestion et élaboration de la stratégie, comment les travaux de Vygotski et de ses suiveurs permettent de faire progresser les analyses et de proposer de nouvelles directions de recherche.

Jean Pralong propose d'appliquer l'approche par l'activité à la recherche sur les carrières. Il suggère que la TA permet de dépasser l'opposition, désormais bien établie, entre d'une part déterminismes individuels et carrière subjective et, d'autre part, déterminismes organisationnels et carrière objective. Il montre que la référence à la carrière traditionnelle véhiculée par les organisations est utilisée par les acteurs dans leur activité pour structurer leur dialogue intérieur. Les décisions de carrière ne sont donc prises, ni de manière totalement subjective, ni uniquement en fonction des cadres pré-établis. Ce qui se dessine à travers cette étude c'est une carriérologie profane, c'est-à-dire une carriérologie qui rend compte du fait que les acteurs ne croient pas nécessairement à des cadres institutionnels qui s'imposeraient à eux, ni aux directions que ceux-ci semblent tracer, mais que les acteurs utilisent et aménagent en fonction de leurs objectifs propres. Si adhésion il y a, celle-ci est réfléchie et correspond davantage à un désir personnel qu'au sentiment de devoir honorer une obligation que fixerait un schéma pré-établi. Finalement, en partant des travaux de la TA, Pralong propose une approche intégrative combinant approches subjective et objective des carrières qui permet de rendre compte de l'environnement des acteurs mais également de la manière dont ceux-ci l'analysent et déploient en conséquence leurs stratégies personnelles de 
carrière.

Aziza Derujinsky-Laguecir, Anja Kern et Philippe Lorino mobilisent la théorie de l'activité pour éclairer les enjeux théoriques et empiriques de la recherche en contrôle de gestion menée sur les indicateurs de performance. Les auteurs proposent de s'émanciper des approches représentationnelles des outils de gestion qui considèrent ceux-ci comme des 'abrégés du vrai', c'est-àdire comme reflétant la vérité, pour les considérer comme des artefacts impliqués dans des processus d'interprétation. En s'appuyant sur les travaux de Rabardel et de ses collègues s'inscrivant dans la lignée de Vygotski, les auteurs indiquent que les artefacts ne constituent pas des instruments finalisés tant qu'ils ne sont pas utilisés, c'est-à-dire tant qu'ils ne soutiennent pas l'activité $d^{\prime}$ une personne. Cette approche considère les artefacts comme structurant la modalité d'interaction entre les individus et leur environnement, mais elle insiste également sur la nécessité de rendre compte des écarts entre les utilisations effectives d'un artefact et les prévisions du concepteur. L'artefact ne doit pas être compris comme un instrument de contrainte mais bien plutôt comme un espace de possibilités dont certaines seront saisies et d'autres pas, permettant à la fois aux utilisateurs de construire des points de vue distincts (et parfois divergents) et de rechercher des compromis. L'activité est alors définie comme la tension entre les prescriptions formelles portées par les indicateurs de performance et leur appropriation, et plus généralement la tension entre le donné et le créé. C'est cette tension qui constitue la source même du développement et des transformations de l'activité. Alors que la question de l'appropriation des outils de gestion se trouve au cœur des interrogations contemporaines du contrôle de gestion (voir par exemple de Vaujany, 2006), les auteurs montrent que la TA constitue un puissant moyen d'analyse des processus collectifs de cette appropriation.

Dans le domaine du comportement du consommateur, Valérie-Inès de la Ville et Valérie Tartas montrent l'intérêt des travaux de Vygotski et de ses suiveurs pour modéliser le processus de socialisation des consommateurs, et pallier certaines limites inhérentes aux approches habituellement mobilisées en marketing. Elles indiquent que ces dernières ont puisé dans l'œuvre complexe de Piaget l'idée d'une conception linéaire de la socialisation de l'individu qui les conduit à mettre davantage l'accent sur les acquis cognitifs que sur les processus 
culturels et sociaux d'intériorisation des compétences de consommation. La théorie de l'activité permet de rendre compte du développement de l'enfant consommateur en conjuguant à la fois l'influence de l'environnement sur cet enfant et la manière dont celui-ci peut parfois jouer avec les règles qu'on lui impose, les détourner, ou les aménager à sa convenance. C'est l'activité de participation de l'enfant aux activités conjointes de consommation qui devient alors centrale. La socialisation doit être comprise comme le passage d'une participation périphérique à la consommation, en tant qu'observateur ou simple usager du produit, à une participation centrale, en tant que décideur de ses propres pratiques de consommation. A partir de cette analyse, Inès de la Ville et Valérie Tartas mettent en lumière la co-existence de quatre projets scientifiques pour aborder la formation des compétences du consommateur.

Jean Louis Magakian utilise les apports de la TA pour étudier l'élaboration de la stratégie des dirigeants d'entreprises. L'analyse par l'activité permet de rendre compte de celle-ci comme une pratique émergente au cours de laquelle le dirigeant s'efforce de prendre en compte les éléments existants et d'élaborer une décision en s'appuyant sur son activité conversationnelle. En lien avec les travaux considérant la stratégie comme une pratique, Magakian montre que la fabrication de la stratégie est une activité quotidienne, faite de conversations, d'échanges et de bricolages. Ce caractère quotidien de l'activité ne veut pas dire que le processus d'idéation ne peut être managé, mais qu'au contraire le dirigeant doit organiser son environnement afin que les conversations, les réunions et les autres instruments qu'il peut utiliser dans son quotidien lui soient réellement utiles pour penser la meilleure stratégie possible. C'est donc bien une grille d'analyse et d'organisation du management de I'idéation que Magakian propose en partant de la TA.

Le dossier se clôt sur un entretien que Jean Louis Magakian a mené avec Yrjö Engeström. Ce dernier revient sur l'évolution de la théorie de l'activité, sa position actuellement dans le courant dit des approches par les pratiques, présente les travaux actuels de son centre de recherche, le CRADLE à l'Université d'Helsinki, et offre une réflexion sur la position actuelle de la TA dans le champ du management et des perspectives de développement futur qui lui semblent les plus prometteuses. Par manque de place nous ne publions dans ce dossier qu'une partie de cet entretien dont l'intégralité peut être lue et téléchargée sur 
le site du CRADLE Helsinki (http://www.helsinki.fi/cradle/chat.htm).

\section{Références}

Benslimane K. et Leca B. (2010), Le travail institutionnel : Fondements théoriques, défis et perspectives, Management \& Avenir, No. 37 : 53-69.

Blacker F. et Regan S. (2006), Institutional Reform and the Reorganization of Family Support Services, Organization Studies, 27 / 12 : 1843-1861.

Blacker F. et Regan S. (2009), Intentionality, Agency, Change: Practice Theory and Management, Management Learning, 40 / 2 : 161-176

Bronckart J.-P. (1985), Vygotsky, une oeuvre en devenir, in : B. Schneuwly \& J.P. Bronckart (Eds), Vygotsky aujourd'hui, Paris, Delachaux \& Niestlé, 7-21.

Bruner J. (1990), Acts of Meaning. Cambridge, MA : Harvard University Press Clot Y. (dir.), (1999), Avec Vygotski, Paris : La Dispute.

Cole M. (2006) The Fifth Dimension An After-School Program Built on Diversity, Russell Sage Foundation Publications.

Cunliffe A. L. (2001a), Managers as Practical Authors: Reconstructing our Understanding of Management Practice, Journal of Management Studies, Vol. $38: 351-371$.

Cunliffe A. L. (2001b), Everyday Conversations: a Social Poetics of Managing, Concepts and Transformations, 6 / 3 : 295-315.

Cunliffe, A. L., (2002), Social Poetics: a Dialogical Approach to Management Inquiry, Journal of Management Inquiry, Vol. 11 : 128-146.

de La Ville V.I. (1996), " Apprentissages collectifs et structuration de la stratégie dans la jeune entreprise de haute technologie. Etude de cas et éléments de modélisation procédurale ", Thèse de Doctorat en Sciences de Gestion, Université Lyon 3.

de Vaujany F.X. (2006), Pour une théorie de l'appropriation des outils de gestion : vers un dépassement de l'opposition conception-usage, Management et Avenir, $\mathrm{N}^{\circ} 9$ : 109-127.

Donald, M. (1991), Origins of the Modern Mind: Three Stages in the Evolution of Culture and Cognition, Cambridge, MA: Harvard University Press. 
Druid. Lave, J et Wenger E, (1991), Situated Learning: Legitimate Peripheral Participation, Cambridge: Cambridge University Press.

Engeström Y. (1987), Learning by expanding: An activity-theoretical approach to developmental research. Helsinki: Orienta-Konsultit.

C. Eden, et J.-C. Spender (1998, Managerial and organizational cognition: theory, methods and research. London: Sage

Gergen, K. J. ( 1999), An invitation to social construction. Londres: Sage

Gorlsorkhi,D.(Ed.)(2006),La Fabrique de la stratégie .une perspective multidimensionnelle, Paris : Vuibert.

Gorlsorkhi, D. Rouleau L. Seidl D. et Vaara E. (Eds.) (2010), Cambridge Handbook of Strategy as Practice. Cambridge University Press.

Greenwood, R., Suddaby R., et Hinings C.R. (2002), Theorizing change: The Role of Professional associations in the Transfomation of Institutionalized Fields, Academy of Management Journal, 45 / 1 : 58-80

Huault, I. et Leca, B. (2009), Pouvoir et Institutions. Une vision radicale, Revue Française de Gestion, № 193 : 133-149.

Hutchins E. (1995). How a cockpit remembers its speeds, Cognitive Science, 19 : 265- 288

Jarzabkowski P. (2003), Strategic Practices: An Activity Theory Perspective on Continuity and. Change, Journal of Management Studies, 40 / 1 : 22-55.

Jarzawbowski P. (2005), Strategy-as-Practice : An Activity-Based View. London: Sage.

Jarzawbowski P. (2010), An activity-theory approach to Strategy as Practice, in Gorlsorkhi, D., L. Rouleau, D. Seidl et E. Vaara (Eds.) Cambridge Handbook of Strategy as Practice. Cambridge University Press, 127-140.

Laguecir A. (2003), Practices of Cost Planning and Management Tools in NPD process: A Quali-Comparative Approach, Communication, Winter Conférence du réseau

Lawrence T. et Suddaby R. (2006), Institutions and Institutional Work, in Clegg S., Hardy C., Nord W.et Lawrence T. (Eds) Handbook of Organization Studies, London: Sage, 215- 254.

Leontiev, A.N. (1978). Activity, consciousness and personality. NJ: Prentice-Hall Lorino P. (1995), Comptes et récits de la performance: essai sur le pilotage de 
I'entreprise, Les Editions d'Organisation, Paris

Lorino, P. (2006) The Instrumental Genesis of Collective Activity: The Case of an ERP Implementation in a Large Electricity Producer. Communication à la conférence OLKC 2006 (Warwick).

Luria, A. R. (1976), Cognitive development: Its cultural and social foundations. Cambridge: Harvard University Press.

Meyer, J. W. et Jepperson, R. (2000) The "actors" of modern society: the cultural construction of social agency, Sociological Theory, 18 / 1 : 100-120.

Meyer, J. W. \& Rowan, B. (1977). Institutionalized organizations: formal structure as myth and ceremony. American Journal of Sociology, 83: 440-463.

Mutch, A. (2010) Technology, Organization, and Structure-A Morphogenetic Approach Organization Science, 21: 507-520

Nardi, B.A. (1996), Context and Consciousness: Activity Theory and HumanComputer Interaction, Cambridge, MA : The MIT Press.

Nardi, B.A. (2010), My Life as a Night Elf Priest, Michigan University Press. Norman, D.A. (1993), Les artefacts cognitifs, Raisons Pratiques, № $4: 15-34$

Norman, D. A. (1993), Things That Make Us Smart: Defending Human Attributes in the Age of the Machine. Reading, MA: Addison-Wesley Pub.

Powell, W.W. et Colyvas, J. (2008), Microfoundations of Institutional Theory in Greenwood R., Oliver C., Suddaby R. et Sahlin-Andersson K. (Eds) The SAGE Handbook of Organizational Institutionalism, Sage, 276-98.

Schatzki, T. (2005), Peripheral Vision: The Sites of Organizations, Organization Studies, 26 / $3:$ 465-84.

Schatzki, T., (2001), Introduction : Practice Theory, in Schatzki, T., Knorr-Cetina, K., et Von Savigny, E. (Eds.) The Practice Turn in Contemporary Theory. London: Routledge.

Scott, R. W. (2001), Institutions and Organizations, 2nd edn. Thousand Oaks, CA: Sage Shotter, J. (1993), Conversational Realities: Constructing Life Through Language, Thousand Oaks, CA : Sage.

Spender, J.C. 1995, Organizations are activity systems, not merely systems of thought, Advances in Strategic Management, 12 B : 153-174

Spender, J.C. (1996), Organizational knowledge, learning and memory: three concepts in search of a theory, Journal of Organizational Change Management, 
$9 / 1: 63-78$.

Spender, J.C., (1998), The Dynamics of Individual and Organizational Knowledge, in C. Eden et J.C. Spender Managerial and Organizational Cognition: Theory, Methods and Research, Sage Publications, London, 13-39.

Suchman, L.A. (1987), Plans and Situated Action: The Problem of Human Machine Communication, Cambridge, MA : Cambridge University Press.

Vygotski, L.S. (1997), Langage et pensée, Paris : La Dispute.

Wertsch, J.V. (1985), Cultural, Communication, and Cognition: Vygotskian Perspectives. Cambridge University Press. 